\title{
THE DATA DRIVEN SURROGATE MODEL BASED DYNAMIC DESIGN OF AERO- ENGINE FAN SYSTEMS
}

\author{
Yun-Peng Zhu ${ }^{1}$, Jie Yuan ${ }^{2}$, Z Q Lang ${ }^{1}$, C W SchwingshackI ${ }^{2}$, Loic Salles ${ }^{2}$, V Kadirkamanathan ${ }^{1}$ \\ ${ }^{1}$ Department of Automatic Control and Systems Engineering, University of Sheffield, Mappin Street, Sheffield, S1 \\ 3JD, UK \\ ${ }^{2}$ Department of Mechanical Engineering, Imperial College London, SW7 2AZ, London, UK
}

\begin{abstract}
High cycle fatigue failures of fan blade systems due to vibrational loads are of great concern in the design of aero engines, where energy dissipation by the relative frictional motion in the dovetail joints provides the main damping to mitigate the vibrations. The performance of such a frictional damping can be enhanced by suitable coatings. However, the analysis and design of coated joint roots of gas turbine fan blades are computationally expensive due to strong contact friction nonlinearities and also complex physics involved in the dovetail. In this study, a data driven surrogate model, known as the Nonlinear in Parameter AutoRegressive with eXegenous input (NP-ARX) model, is introduced to circumvent the difficulties in the analysis and design of fan systems. The NPARX model is a linear input-output model, where the model coefficients are nonlinear functions of the design parameters of interest, such that the Frequency Response Function (FRF) can be directly obtained and used in the system analysis and design. A simplified fan bladed disc system is considered as the test case. The results show that by using the data driven surrogate model, an efficient and accurate design of aero-engine fan systems can be achieved. The approach is expected to be extended to solve the analysis and design problems of many other complex systems.
\end{abstract}

Keywords: Fan blade system; Contact friction; Dry film lubricant coating; Data driven; Surrogate model; Design

THE LIST OF FIGURE AND TABLE CAPTIONS

FIGURE 1: ILLUSTRATION OF THE BLADE SYSTEM

WITH ROOT COATINGS

FIGURE 2: THE FRICTION NONLINEARITY

FIGURE 3: EFFECTS OF FRICTION DESIGN PARAMETERS

ON SYSTEM DYNAMICS

FIGURE 4: THE FRICTION NONLINEARITY UNDER

DIFFERENT DESIGN PARAMETERS

FIGURE 5: FRAMEWORK OF THE DATA DRIVEN

SURROGATE MODEL BASED DESIGN

FIGURE 6: THE DISCREPANCIES OF THE 2-

DIMENSIONAL DISTRIBUTION

FIGURE 7: THE UD SAMPLING POINTS FOR THE BLADE

SYSTEM DESIGN

FIGURE 8: THE FIRST AND THE THIRD VIBRATION

MODE OF THE BLADE SYSTEM

FIGURE 9: MODEL PREDICTION OF BLADE SYSTEM

RESPONSES UNDER THE FIRST ORDER MODE
FIGURE 10: MODEL PREDICTION OF BLADE SYSTEM

RESPONSES UNDER THE THIRD ORDER MODE

FIGURE 11: VALIDATION OF THE DESIGN RESULTS

Tab.1 The model prediction results for the first order mode

Tab.2 The model prediction results for the third order mode

Tab. A The coefficients of the surrogate model for the first vibration mode

\section{INTRODUCTION}

Fan blade system has been widely regarded as one of the fundamental factors that determine the overall performance of a modern aero-engine. They are not just decisive on the level of maximum thrust generated to propel an aircraft but also critical on structural efficiency and durability of engines $[1,2,6]$.

In the design process of bladed disc systems, high cycle fatigue failures induced by vibrational loads are of great concern to aero-engine manufacturers. Frictional damping is widely used in gas turbine engine to mitigate vibrations through energy dissipation by exploiting the contact surfaces such as under platform damper in turbines. Krack et al. [3] recently gives a detailed review of the utilization of the dry friction damping to mitigate the structural vibrations of the mechanical joints in gas turbine engines. Sun et al. [5] showed numerically that the vibration of the blades on the integrated bladed-disk can be effectively controlled by the parameters of the frictional ring damper. In terms of fan blade system, the major contribution is also from the frictional damping in the dovetail joint [3-5]. This way of energy dissipation in the fan system can be optimized by applying suitable dry film lubricant coatings on the blade root as it can effectively change the contact conditions by reducing the frictional coefficient [6]. The main purpose of applying the coating is to address the problem with fretting fatigue by reducing the tangential contact stress in joint interface [7]. The solid lubricant Molydag 254 is mostly used, which consists of MoS2 powder, carbon and other solid lubricants in a thermosetting phenolic resin [6].

The present study is focused on the assessment of the effects of this coating on the vibrational behavior. However, the analysis and design of gas turbine fan blades with dovetail joint are computationally expensive due to strong nonlinearities and complex physics involved in the dovetail [8]. Harmonic balance method has been used to determine the steady state response of fan systems with localized contact friction nonlinearities, which can reduce the computational time by the ten or hundred times 
comparing to the traditional time domain methods such as Newmark method [3,9]. Component model synthesis techniques are also widely used for model order reduction to make the computation of a large-scale assembly feasible $[3,8]$. Yuan et al. [9] compared different reduced order modelling techniques for dynamic analysis of jointed structures with localized contact nonlinearities. Even with the use of these classical reduced order modelling techniques, the computational cost for a large scale system remains extremely high due to the intensive contact frictional interface and the requirement of a dense mesh [10]. The comparisons indicate that there is a high demand in industries to develop efficient methods to improve the design of complicated fan systems.

Recently, analyses of system dynamics based on data driven surrogate models have shown great advantages in investigating complex systems [11-13]. For example, Bruntona et al. [11] combined sparsity techniques and machine learning with nonlinear dynamical systems to derive governing equations from noisy measurement data. Both the Lorenz system and fluid vortex shedding behind an obstacle were studied based on an achieved data driven model. Billings [13] introduced a general type of nonlinear model known as the Nonlienar AutoRegressive with eXegenous input (NARX) model, which can be identified by using the NARMAX approach and then be used to study the system using frequency domain analysis techniques [14, 15].

In this study, a data driven surrogate model, known as the Nonlinear in Parameter ARX (NP-ARX) model, is introduced to circumvent the difficulties in the analysis and design of fan systems. The NP-ARX model is a linear input-output model, where the model coefficients are nonlinear functions of the design parameters of interest. Compared with other surrogate models such as, for example, the neural network model [16] and the NARX model [13], the NP-ARX model is more physically meaningful since the design parameters of interests explicitly appear as coefficients in the model, and the Frequency Response Function (FRF) can be directly obtained from the NP-ARX model for the system analysis and design [14].

A simplified fan bladed disc system with contact friction Jenkins elements are considered as the test case. The effect of the coating on the contact surface is represented by the contact parameters in the Jenkins element based on the experiments conducted in $[6,7]$. The NP-ARX model based design of a fan system involves tree steps which are (1) Collection of the high fidelity model/experiment generated system input and output data under different choices of the design parameters; (2) Determination of the NP-ARX model of the gas turbine fan blade with coated roots; and (3) Design the root coating parameters using the FRF of the NP-ARX model.

\section{THE FAN BLADE SYSTEM FOR DESIGN}

\subsection{The aero-engine fan blade with root coatings}

A sector of fan blade system with coated dovetail joints is shown in Fig.1, where the friction between the blade and disc in the dovetail joints is used to mitigate vibrations through energy dissipation. The blade system can be simplified to a cantilever beam with a $1 \mathrm{D}$ frictional element shown in Fig.1, where the system design parameters of interest and their effects on the system dynamic characteristics are illustrated. This simplified system of the fan blade is used for a case study in this paper. In Fig.1, $F(t)$ and $y(t)$ are the input force and the output displacement of the blade system, respectively; The friction coefficient, contact stiffness and preloading are denoted as $\mu, K_{T}$ and $N_{0}$, respectively, representing the design parameters of the root coating and related to the frictional damping of the system.

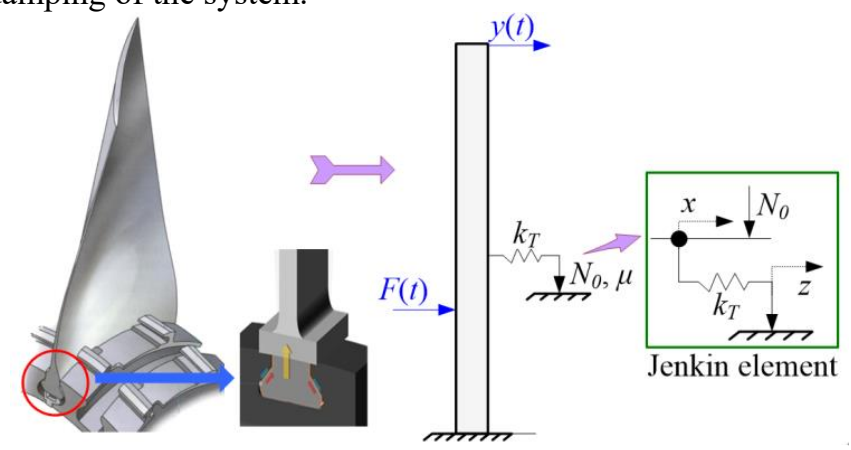

FIGURE 1: ILLUSTRATION OF THE BLADE SYSTEM WITH ROOT COATINGS

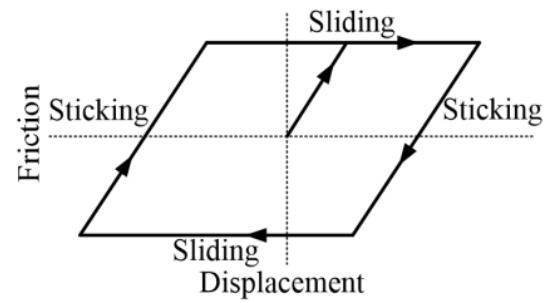

FIGURE 2: THE FRICTION NONLINEARITY

The contact friction characteristic is represented by using the Jenkin element model shown in Fig.1, where $x$ and $z$ are the internal variables for sliding position [5]. There are two contact conditions in the Jenkin element model which are sticking and sliding. In a sticking condition, the contact force is linear elastic and proportional to the tangential relative displacement while in a sliding condition, the contact node starts to slide between the contact surfaces in the dovetail joints as illustrated by the hysteresis loop in Fig.2. The nonlinear contact friction is a constant of the value of $\mu N_{0}$.

The equation of motion for this nonlinear system with contact friction force can be written as

$$
\mathbf{M} \ddot{\mathbf{y}}(t)+\mathbf{C} \dot{\mathbf{y}}(t)+\mathbf{K y}(t)+\mathbf{F}_{n l}(\dot{\mathbf{y}}(t), \mathbf{y}(t))=\mathbf{F}(t)
$$

where $\mathbf{M}, \mathbf{C}, \mathbf{K}$ are the mass, damping, and stiffness matrices; $\mathbf{F}(t)$ represents the external forces and $\mathbf{F}_{n l}($.$) is$ the nonlinear contact force which is dependent on the relative displacement in the joint interface.

The nonlinear vibration of this simplified blade system is analysed by using the FOrce Response SuitE (FROSE) code 
developed by Vibration Technology Centre at Imperial College London. This is a frequency domain solver and based on the Multiple Harmonic Balance Method (MHBM) [5, 17]. The MHBM based solution to the model output responses can be represented using a truncated Fourier series. The unknowns in Eq.(1) can be expressed in the frequency domain as:

$\mathbf{y}(t)=\mathbf{Q}_{0}+\sum_{j=1}^{n}\left[\mathbf{Q}_{j}^{c} \cos \left(m_{j} \omega t\right)+\mathbf{Q}_{j}^{s} \sin \left(m_{j} \omega t\right)\right]$

where $n$ is the number of the harmonics; $\omega$ is the excitation frequency; $\mathbf{Q}_{0}, \mathbf{Q}_{j}^{c}$ and $\mathbf{Q}_{j}^{s}$ are the cosine and sine harmonic coefficients, which can be determined by substituting equation (2) into equation (1) and solving:

$\tilde{\mathbf{Z}} \mathbf{Q}+\tilde{\mathbf{F}}_{n l}(\mathbf{Q})-\tilde{\mathbf{F}}=0$

where $\mathbf{Q}=\left[\mathbf{Q}_{0}, \mathbf{Q}_{1}^{c}, \mathbf{Q}_{1}^{s}, \ldots, \mathbf{Q}_{n}^{c}, \mathbf{Q}_{n}^{s}\right]^{\mathrm{T}}$ is the vector of harmonic coefficients; $\tilde{\mathbf{Z}}$ is the dynamic stiffness matrix in the frequency domain [5, 17], $\tilde{\mathbf{F}}_{n l}($.$) and \tilde{\mathbf{F}}$ are the vectors of nonlinear force and external excitation in the frequency domain $[5,17]$.

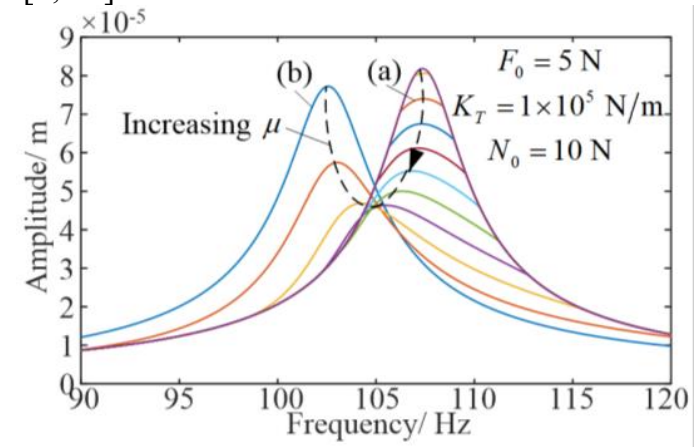

FIGURE 3: EFFECTS OF FRICTION DESIGN PARAMETERS ON SYSTEM DYNAMICS

The level of dissipating nonlinearity in the blade system due to the contact friction is dependent on the parameters of $\mu, K_{T}, N_{0}$ and the input force magnitude $F_{0}$, and the effects of these design parameters on the system output responses. For example, the effect of an increase of the friction coefficient $\mu$ from 0.1 to $0.9[6,7]$ with a fixed $K_{T}, N_{0}$ and $F_{0}$, are as illustrated in Fig.3.

It can be seen from Fig. 3 that the friction can significantly affect the system output characteristics such as the resonance and amplitude. The variation of the dynamic characteristics of the blade system can be explained by changing nonlinear friction forces shown in Fig.4 which shows the variation of the frictional force with the relative displacement over one period. The area of the hysteretic loop is the energy dissipation by contact friction in a vibrational period. If the friction coefficient is small like, for example, the curve (a) in Fig.3, the friction force is shown in Fig.4 (a) then the equivalent stiffness $k_{a}$ is large and the resonant frequency is high. If the friction coefficient is large like, for example, the curve (b) in Fig.3, the friction force is shown in Fig.4 (b) then the equivalent stiffness $k_{b}$ is small and the resonant frequency is low. The level of the amplitude in Fig. 3 depends on the ratio of the energy dissipation by the contact friction shown in the area in Fig. 4 to the input energy from the external excitation.

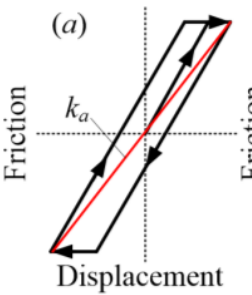

(b)

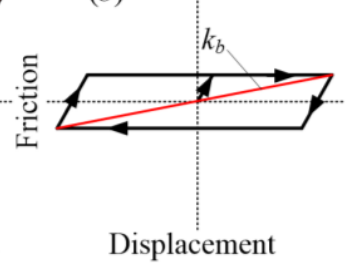

FIGURE 4: THE FRICTION NONLINEARITY UNDER DIFFERENT DESIGN PARAMETERS

It is worth noting that analysis and design of a beam with 1D friction element is not time consuming based on a physical model as presented above [9], but when it comes to the 3D high fidelity fan blade system, the analysis and design of the blade system with coated roots would become extremely computationally expensive due to strong nonlinearities let alone the complex physics involved in the dovetail. As such, a data driven approach is investigated herein to reduce the associated computational cost. The surrogate model based on NP-ARX model identified from the input and output data of the simplified blade system will also be used for the design of the blade system with coated roots.

\subsection{Dynamic design using data driven models}

The process of the data driven surrogate model based design is shown in Fig.5, where in this study, a linear inputoutput discrete time ARX model is identified with model coefficients being a nonlinear function of the system design parameters. Therefore, the linear frequency domain design approaches can be applied based on the data driven model to conduct the design of the fan blade system.

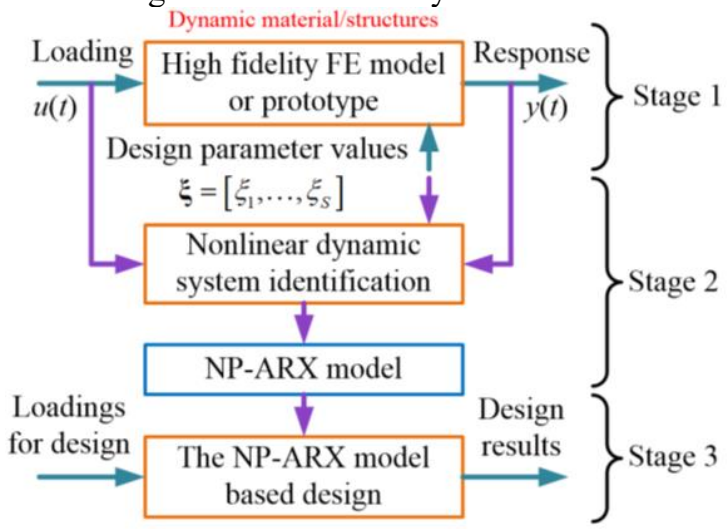

FIGURE 5: FRAMEWORK OF THE DATA DRIVEN SURROGATE MODEL BASED DESIGN

According to Fig.5, in Stage 1, the input and output data from a high fidelity Finite Element (FE) simulation are periodic signals, where the input is the aerodynamic force loaded on the 
fan blade, and the output is the displacement on the blade tip. The design parameters are the parameters selected from a design space to train the NP-ARX model, and three different distributions for selecting the design parameters are considered in this step. In Stage 2, a new frequency domain identification approach based on the Forward Regression Orthogonal Least Squares (FROLS) algorithm [13] is applied to build an NPARX model representing the relationship between the output displacement of the blade tip, the input aerodynamic force loading, and all design parameters of interest. As a result, the effects of the input force and design parameters on the system displacement output can be studied, and the dissipation of the vibration energy can be evaluated based on the NP-ARX model. An optimal design problem is then formulated in Stage 3 based on the FRF of the NP-ARX model. The design results are then numerically verified by performing additional FE model tests to demonstrate the performance of the data driven surrogate model based system design. Details of each stage are explained as follows.

\section{Stage 1: Data collection}

In this stage, the input and output data are collected from a high fidelity FE model or prototypes. In order to identify a surrogate model with design parameters of interests, changing values of each design parameter are also required. The data collection follows two steps. Firstly, the system input and the range of variations of the design parameters are specified. Secondly, the values of design parameters for training are determined using an experimental design approach such as the orthogonal design, the Uniform Design (UD) [18] or the Latin Hypercube Sampling (LHS) [19], etc.

\section{Stage 2: System identification}

The NP-ARX model is identified in this stage by using the data collected in Stage 1 to represent the dynamic behaviours of the system for design. The NP-ARX can generally be written as

$$
y(k)=\sum_{k_{y}=1}^{K} \theta_{\left(k_{y}, 0\right)}(\xi) y\left(k-k_{y}\right)+\sum_{k_{u}=1}^{K} \theta_{\left(0, k_{u}\right)}(\xi) u\left(k-k_{u}\right)
$$

where $k$ represents discrete time; $u($.$) and y($.$) are the$ system input and output, respectively; $K$ is an integer; $\theta_{\left(n_{y}, n_{u}\right)}(\xi)$ with $n_{y}, n_{u}=0, \ldots, K ; n_{y}+n_{u} \geq 1$ are the model coefficients, representing a nonlinear function of the design parameters $\xi=\left[\xi_{1}, \ldots, \xi_{S}\right]$ with $S$ being an integer.

It is known that many time domain identification approaches have been developed to build the data driven model with design parameters based on the NARXMAX approaches [20,21]. However, a random excitation is required in these methods and when the input forces are harmonic excitations, the algorithm becomes heavily redundant and computationally expensive due to a large amount of repetitive periodical time domain signals. In order to address this issue, in this study, a frequency domain based identification algorithm is introduced to determine the NP-ARX of the system.

\section{Stage 3: System design}

Noting that the NP-ARX model (4) is a linear input output model, the analysis and design issues can be addressed in the frequency domain by using the linear Frequency Response Function (FRF) [22]

$$
H(\mathrm{j} \omega)=\frac{\sum_{k_{u}=1}^{K} \theta_{\left(0, k_{u}\right)}(\xi) \exp \left(-\mathrm{j} k_{u} \omega \Delta t\right)}{1-\sum_{k_{y}=1}^{K} \theta_{\left(k_{y}, 0\right)}(\xi) \exp \left(-\mathrm{j} k_{y} \omega \Delta t\right)}
$$

where $\omega=2 \pi f$ is the angular frequency, and $\Delta t$ is the sampling time.

In the following, the design of the fan blade with coated roots will be conducted following the three stages introduced above based on the FE model provided by Imperial College.

\section{THE SURROGATE MODEL BASED DESIGN OF THE FAN BLADE SYSTEM}

\subsection{Determination of design parameters for training}

In this work, the input excitations are harmonic forces of

$F(t)=F_{0} \cos (2 \pi f t)$

over the range of frequency $f \in[95,120] \mathrm{Hz}$, in which the resonance of the blade varies with the design parameter values.

$\mu, K_{T}$ and $N_{0}$ are the design parameters for the root coating. As the input force magnitude also affects the friction force in the blade system, the input force magnitude $F_{0}$ is also taken as a design parameter. Consequently, the unit signal $u(t)=\cos (2 \pi f t)$ is applied for the NP-ARX model identification, and the design parameters of interest are

$\xi=\left[F_{0}, \mu, K_{T}, N_{0}\right]$

Based on the previous experimental studies in $[6,7]$, the considered ranges of values of the design parameters of interest are taken as

$F_{0} \in[1,16] \mathrm{N} ; \mu \in[0.05,0.95]$

$K_{T} \in[5,15] \times 10^{4} \mathrm{~N} / \mathrm{mm} ; N_{0} \in[1,20] \mathrm{N}$

Because there are 4 design parameters, if each parameter has 5 different values to test, there is a total number of 625 tests for a full scale design, which is a large amount of tests for either FE simulation or experiment. In order to identify the NPARX model by using less testings, in this study, the UD method is applied to determine $N_{t}$ number of tests with the design parameters uniformly distributed over the space as formulated by (8). 
The UD method was developed based on the geometric sense of discrepancy defined as follows

Definition [18]: Let $\boldsymbol{\Xi}=\left\{\mathbf{x}_{k}, k=1, \ldots, n\right\}$ be a set of points on a $s$-dimensional unit cube $C^{s}$, where $\mathbf{x}_{k}=$ $\left[x_{k_{1}}, \ldots, x_{k_{s}}\right]^{\mathrm{T}}$ is an $s$-dimensional column vector. For any $\boldsymbol{\gamma} \in C^{s}$, let $N(\boldsymbol{\gamma}, \boldsymbol{\Xi})$ be the number of points satisfying $\mathbf{x}_{k} \leq \boldsymbol{\gamma}$, which means $x_{k_{i}} \leq \gamma_{i}, i=1, \ldots, s$. Then

$D(n, \boldsymbol{\Xi})=\sup _{\gamma \in C^{s}}\left|\frac{N(\boldsymbol{\gamma}, \boldsymbol{\Xi})}{n}-v([\mathbf{0}, \boldsymbol{\gamma}])\right|$

is defined as the discrepancy of $\boldsymbol{\Xi}$, where $v([\mathbf{0}, \boldsymbol{\gamma}])$ $=\gamma_{1} \times \cdots \times \gamma_{s}$ denotes the volume of the rectangle $[\mathbf{0}, \boldsymbol{\gamma}]$.

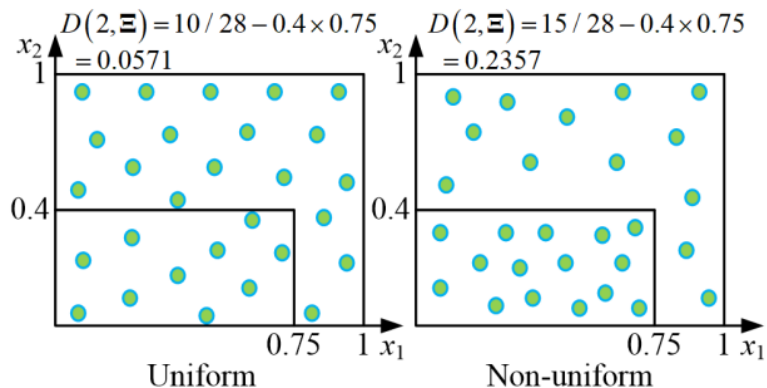

FIGURE 6: THE DISCREPANCIES OF THE 2-DIMENSIONAL DISTRIBUTION

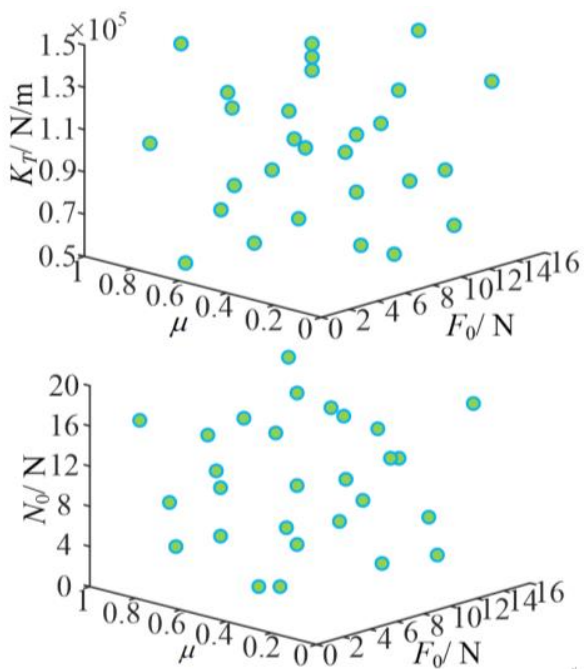

FIGURE 7: THE UD SAMPLING POINTS FOR THE BLADE SYSTEM DESIGN

The basic idea of the UD method is that when the sampling points in $\boldsymbol{\Xi}$ are uniformly distributed in the design space $C^{s}$, the ratio $N(\boldsymbol{\gamma}, \boldsymbol{\Xi}) / n$ is close to the volume of the rectangle $[\mathbf{0}, \boldsymbol{\gamma}]$, and the discrepancy defined in (9) can be used to measure the uniformity of the distribution. For example, a 2- dimensional distribution in $C^{2}$ is shown in Fig.6 to illustrate the measurement of the discrepancy.

The UD method can be implemented by minimizing the maximum discrepancy for all possible rectangle $[\mathbf{0}, \gamma]$, and many methods have been proposed to solve this problem. In this study, the Threshold Accepting Method (TAM) [23] is applied to find $N_{t}=28$ UD sampling points in the 4dimensional design parameter space. The results can be directly achieved by using the TAM algorithm toolbox provided on the website [24] and shown in Fig.7.

Then the system input and output spectrum in the frequency range of $f \in[95,120] \mathrm{Hz}$ under these $N_{t}=28$ training parameters are collected for the identification of the surrogate model.

Remark: It is worth noting that many experimental design approaches can be used to guarantee a uniform distribution of the design parameters. For example, the well-known LHS method can also be applied, where the uniformity is defined in a statistic sense, and has been proven closely related to the UD method under specific conditions $[25,26]$.

\subsection{Identification of the surrogate model}

\subsubsection{The model identification algorithm}

According to 3.1, the unit input spectra $U(\mathrm{j} \omega)$ and the output spectrum $Y(\mathrm{j} \omega)$ for the $N_{t}=28$ sets of training parameters as shown in Fig.7 are collected over the frequency range of $\omega=2 \pi f, f \in[95,120] \mathrm{Hz}$.

Represent the functions of design parameters in the NPARX model (4) by a polynomial of the following form

$\theta_{\left(n_{y}, n_{u}\right)}(\xi)=\sum_{i_{1}, \ldots, i_{s}=0}^{I} \varphi_{i_{1}, \ldots, i_{s}}^{\left(n_{y}, n_{u}\right)} \prod_{r=1}^{s} \xi_{r}^{i_{r}}$

where $n_{y}, n_{u}=0, \ldots, K$ and $\varphi_{i_{1}, \ldots, i_{s}}^{\left(n_{y}, n_{u}\right)}$ are the coefficients of the polynomial.

Thus, the NP-ARX model can be written as

$$
\begin{gathered}
y(k)=\sum_{k_{y}=1}^{K} \sum_{i_{1}, \ldots, i_{s}=0}^{I} \varphi_{i_{1}, \ldots, i_{s}}^{\left(k_{y}, 0\right)}\left(\prod_{r=1}^{s} \xi_{r}^{i_{r}}\right) y\left(k-k_{y}\right) \\
+\sum_{k_{u}=1}^{K} \sum_{i_{1}, \ldots, i_{s}=0}^{I} \varphi_{i_{1}, \ldots, i_{s}}^{\left(0, k_{u}\right)}\left(\prod_{r=1}^{s} \xi_{r}^{i_{r}}\right) u\left(k-k_{u}\right)
\end{gathered}
$$

and by applying the Fourier Transform on both sides of (11), the frequency domain representation of the NP-ARX model can be written as 


$$
\begin{gathered}
Y(\mathrm{j} \omega)=\sum_{k_{y}=1 i_{1}, \ldots, i_{s}=0}^{K} \sum_{i_{1}, \ldots, i_{s}}^{I}\left(\prod_{r=1}^{s} \xi_{r}^{k_{r}}\right) Y(\mathrm{j} \omega) \exp \left(-\mathrm{j} k_{y} \omega \Delta t\right) \\
+\sum_{k_{u}=1}^{K} \sum_{i_{1}, \ldots, i_{s}=0}^{I} \varphi_{i_{1}, \ldots, i_{s}}^{\left(0, k_{u}\right)}\left(\prod_{r=1}^{s} \xi_{r}^{i_{r}}\right) U(\mathrm{j} \omega) \exp \left(-\mathrm{j} k_{u} \omega \Delta t\right) \\
\quad \text { In equation (12), } \quad\left(\prod_{r=1}^{s} \xi_{r}^{i_{r}}\right) Y(\mathrm{j} \omega) \exp \left(-\mathrm{j} k_{y} \omega \Delta t\right) \text { and }
\end{gathered}
$$

$\left(\prod_{r=1}^{s} \xi_{r}{ }^{i_{r}}\right) U(\mathrm{j} \omega) \exp \left(-\mathrm{j} k_{u} \omega \Delta t\right)$ are the terms of the NP-ARX model to be selected, and $\varphi_{i_{1}, \ldots, i_{s}}^{\left(n_{y}, n_{u}\right)}$ represent the coefficients of the model to be identified.

By using the system input, output, and the values of the design parameters for model training, the NP-ARX model (4) of the blade system with coated roots is identified by using a newly proposed frequency domain approach based on the Forward Regression Orthogonal Least Square (FROLS) as

\begin{tabular}{|c|c|}
\hline Step 1: & $\begin{array}{l}\text { Collect input spectrum } U^{(n)}\left(\mathrm{j} \omega_{k}\right) \text { and } \\
\text { output spectrum } Y^{(n)}\left(\mathrm{j} \omega_{k}\right) \text { where } n=1, \ldots, N_{t} \\
\text { and } \omega_{k} \quad k=1, \ldots, N_{d} \text { represent } N_{d} \text { different } \\
\text { frequencies. The frequency domain representation } \\
\text { of the NP-ARX model to be identified can be } \\
\text { written in a matrix form as } \\
\overline{\mathbf{Y}}=\overline{\mathbf{P}} \boldsymbol{\Psi} \\
\text { with } \\
\overline{\mathbf{Y}}=\left[\begin{array}{l}\operatorname{Re}(\mathbf{Y}) \\
\operatorname{Im}(\mathbf{Y})\end{array}\right], \overline{\mathbf{P}}=\left[\begin{array}{l}\operatorname{Re}(\mathbf{P}) \\
\operatorname{Im}(\mathbf{P})\end{array}\right] \\
\text { where } \mathbf{Y} \text { is the column vector of the output } \\
\text { spectrum, } \mathbf{P} \text { is an } N_{t} N_{d} \times M \text { matrix contain- } \\
\text { ing all candidate terms in the system frequency } \\
\text { domain representation and } \boldsymbol{\Psi} \text { is the vector of } \\
\text { the model coefficients. }\end{array}$ \\
\hline & $\begin{array}{l}\text { Compute the } m{ }^{\text {th }}(i=1, \cdots, M-s+2) \\
\text { term's Error Reduction Ratio (ERR) as } \\
E R R_{m}=\frac{\left\langle\mathbf{w}_{m}^{(s-1)}, \overline{\mathbf{Y}}\right\rangle^{2}}{\left\langle\mathbf{w}_{m}^{(s-1)}, \mathbf{w}_{m}^{(s-1)}\right\rangle\langle\overline{\mathbf{Y}}, \overline{\mathbf{Y}}\rangle} \\
\text { of the } M-s+2 \text { remaining candidate terms } \\
\text { from } \mathbf{P} \text {, where } \mathbf{w}_{m}^{(s-1)} \text { is the } s-1 \text { th } \\
\text { orthogonalisation of the } m \text { th term from the }\end{array}$ \\
\hline
\end{tabular}
below.

\begin{tabular}{|c|c|}
\hline & $\begin{array}{l}\text { remaining candidate terms given as } \\
\mathbf{w}_{m}^{(s-1)}=\mathbf{p}_{m}-\sum_{r=1}^{s-1} \frac{\left\langle\mathbf{p}_{m}, \mathbf{w}_{r}\right\rangle}{\left\langle\mathbf{w}_{r}, \mathbf{w}_{r}\right\rangle} \mathbf{w}_{r} \\
\text { with } \mathbf{p}_{m} \text { being the } m^{\text {th }} \text { column vector of the } \\
\text { remaining unselected candidate terms in } \overline{\mathbf{P}} \text {, } \\
\langle\cdot, \cdot\rangle \text { denotes the inner product of vectors with } \\
\left\langle\mathbf{v}_{1}, \mathbf{v}_{2}\right\rangle=\mathbf{v}_{1}^{\mathrm{T}} \mathbf{v}_{2} \text {, and } \mathbf{v}_{1}, \mathbf{v}_{2} \text { are column vectors. } \\
\text { The term with the largest ERR among the } \\
M-s+2 \text { terms in this step is the } s-1^{\text {th }} \text { term of } \\
\text { the identified model, where the largest ERR value } \\
\text { is denoted as } E R R^{(s-1)} \text { and let } \\
\mathbf{w}_{s-1}=\mathbf{w}_{l_{s-1}}^{(s-1)}\end{array}$ \\
\hline Step s+1: & $\begin{array}{l}\text { Step } S \text { terminated at the } S_{0} \text { step when } \\
\eta=1-\sum_{i=1}^{S_{0}-1} E R R^{(i)} \leq \rho \\
\text { where } \rho \text { is a pre-specified threshold. } \\
\text { The identified NP-ARX model is the linear } \\
\text { combination of the } S_{0}-1 \text { significant terms and } \\
\text { the corresponding coefficients can be evaluated } \\
\text { by using the least squares method. }\end{array}$ \\
\hline
\end{tabular}

\section{Algorithm: The frequency identification algorithm}

The NP-ARX model can be validated by using the Model Predicted Output (MPO) test, to ensure the system output responses under any inputs of interest can be predicted under any initial conditions.

In the following, the first and the third vibration modes of the blade system will be considered to demonstrate how the identification algorithm can be used to determine a lower and a higher order vibration mode of the system, respectively. The two vibration modes are shown in Fig. 8.

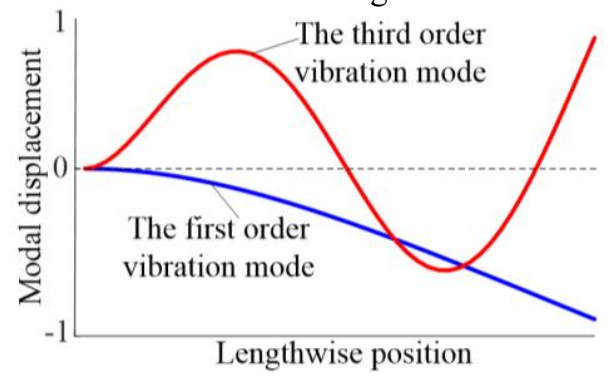

FIGURE 8: THE FIRST AND THE THIRD VIBRATION MODE OF THE BLADE SYSTEM

\subsubsection{The NP-ARX model representing the first order mode}

For a blade system with coated roots, the NP-ARX model representing the first order vibration mode has been identified 
using the above algorithm and data generated in Section 3.1 under the sampling frequency of $f_{s}=1 / \Delta t=1024 \mathrm{~Hz}$ as

$$
\begin{aligned}
& y(k)=\theta_{(1,0)}(\xi) y(k-1)+\theta_{(2,0)}(\xi) y(k-2) \\
& \quad+\theta_{(0,1)}(\xi) u(k-1)+\theta_{(0,2)}(\xi) u(k-2) \\
& \quad+\theta_{(0,3)}(\xi) u(k-3)
\end{aligned}
$$

where

$$
\theta_{\left(n_{y}, n_{u}\right)}(\xi)=\sum_{i_{1}, \ldots, i_{4}=0}^{2} \varphi_{i_{1}, \ldots, i_{4}}^{\left(n_{y}, n_{4}\right)} F_{0}^{i_{1}} \mu^{i_{2}} K_{T}^{i_{3}} N_{0}^{i_{4}}
$$

where the coefficient values are shown in Appendix.

The prediction error as defined by

$\eta=\frac{1}{51} \sum_{i=1}^{51} \frac{|| Y_{\mathrm{P}}\left(\mathrm{j} 2 \pi f_{i}\right)|-| Y_{\mathrm{T}}\left(\mathrm{j} 2 \pi f_{i}\right)||}{\left|Y_{\mathrm{T}}\left(\mathrm{j} 2 \pi f_{i}\right)\right|}$

is used to evaluate the performance of the identified model where the subscript "P" and " $T$ " represent the prediction and the true results, respectively. $f_{i}, i=1, \ldots, 51$ are 51 frequencies over the design frequency range of interest taken as $f_{i}=\{95: 0.5: 120\} \mathrm{Hz}$. The validation of the identified NP-

ARX model is conducted by using four different design parameter sets (a)-(d), and the validation results are shown in Tab.1 and Fig.9.

Tab.1 The model prediction results for the first order mode

\begin{tabular}{ccc}
\hline & Parameter values & Prediction errors \\
\hline (a) & $F_{0}=1 \mathrm{~N}, \mu=0.32$, & \\
& $K_{T}=7.963 \times 10^{4} \mathrm{~N} / \mathrm{m}, N_{0}=8.741 \mathrm{~N}$ & $\eta=6.037 \%$ \\
(b) & $F_{0}=10.444 \mathrm{~N}, \mu=0.95$, & \\
& $K_{T}=1.093 \times 10^{5} \mathrm{~N} / \mathrm{m}, N_{0}=9.444 \mathrm{~N}$ & $\eta=8.417 \%$ \\
(c) & $F_{0}=12.111 \mathrm{~N}, \mu=0.7926$, & \\
& $K_{T}=1.019 \times 10^{5} \mathrm{~N} / \mathrm{m}, N_{0}=14.37 \mathrm{~N}$ & $\eta=6.245 \%$ \\
(d) & $K_{T}=1.204 \times 10^{5} \mathrm{~N} / \mathrm{m}, N_{0}=12.259 \mathrm{~N}$ & $\eta=4.698 \%$ \\
\hline
\end{tabular}

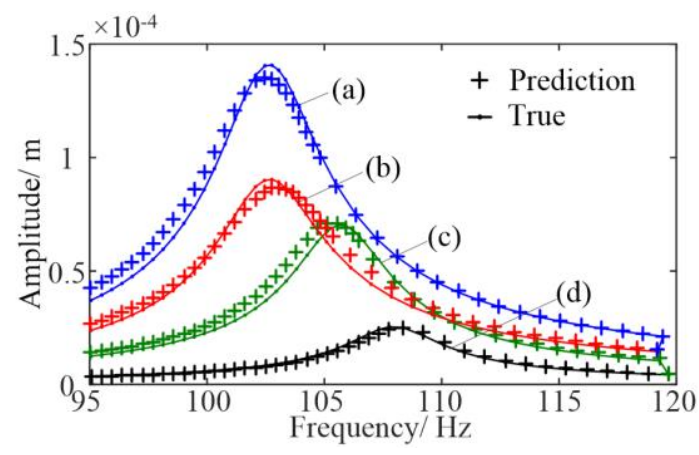

FIGURE 9: MODEL PREDICTION OF BLADE SYSTEM RESPONSES UNDER THE FIRST ORDER MODE

It can be seen from Fig.9 that the identified model (16) can accurately predict the system output response under different design parameters. The model can, therefore, be applied for the system design.

\subsubsection{The NP-ARX model representing the third order mode}

The NP-ARX model representing the third order vibration mode of the blade system with coated roots has been identified using the same algorithm and the training data in Section 3.1 under the sampling frequency of $f_{s}=1 / \Delta t=1024 \mathrm{~Hz}$. The model takes the same structure as (19). Similarly, the identified model is validated by using the design parameters in Tab. 2 over the third order frequency range of interest $\left\{f_{1}, \cdots, f_{51}\right\}$ $=\{275: 0.5: 300\} \mathrm{Hz}$. Equation (21) is used to evaluate the performance of the identified model. the results are shown in Tab. 2 and Fig. 10.

It can be seen from Fig.10 that the identified NP-ARX model can also provide accurate prediction results on higher order modes of the blade system.

Compared to the first order mode vibration, it can be observed from Figs.9 and 10 that the friction design parameters including $\mu, K_{T}$ and $N_{0}$ have much less effects on the output performance of the blade system. This is because in higher mode vibrations, the system responses are relatively less significant so that the friction effects are not as strong as illustrated in Fig.4.

Tab.2 The model prediction results for the third order mode

\begin{tabular}{ccc}
\hline & Parameter values & Prediction errors \\
\hline (a) & $F_{0}=1.241 \mathrm{~N}, \mu=0.197$, & \\
& $K_{T}=9.31 \times 10^{4} \mathrm{~N} / \mathrm{m}, N_{0}=9.379 \mathrm{~N}$ & $\eta=5.311 \%$ \\
(b) & $F_{0}=2.207 \mathrm{~N}, \mu=0.417$, & \\
& $K_{T}=7.586 \times 10^{4} \mathrm{~N} / \mathrm{m}, N_{0}=7.517 \mathrm{~N}$ & $\eta=3.467 \%$ \\
(c) & $K_{0}=4.379 \mathrm{~N}, \mu=0.486$, & \\
& $K_{T}=6.379 \times 10^{4} \mathrm{~N} / \mathrm{m}, N_{0}=8.138 \mathrm{~N}$ & $\eta=3.380 \%$ \\
(d) & $K_{T}=7.414 \times 10^{4} \mathrm{~N} / \mathrm{m}, N_{0}=3.483 \mathrm{~N}$ & $\eta=2.521 \%$ \\
\hline
\end{tabular}

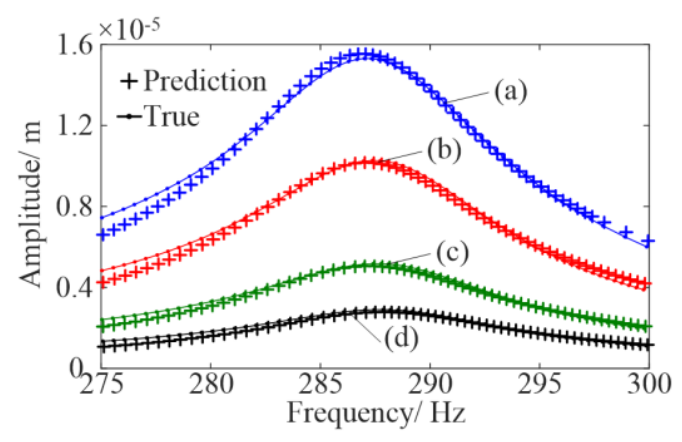

FIGURE 10: MODEL PREDICTION OF BLADE SYSTEM RESPONSES UNDER THE THIRD ORDER MODE

Consequently, the surrogate model based design for the blade system with coated roots will be conducted only based on the first vibration mode in following. 


\subsection{The surrogate model based design}

The design of the fan blade system is conducted by using the FRF of the identified surrogate model (19).

Consider the case where the aim of design is to determine a set of parameters $\xi=\left[F_{0}, \mu, K_{T}, N_{0}\right]$ such that the resonant frequency of the system is $f_{r d}=106 \mathrm{~Hz}$, and the system output magnitude at resonance $\left|Y\left(\mathrm{j} f_{r d}\right)\right| \leq 3.5 \times 10^{-5} \mathrm{~m}$.

According to equation (5), the FRF of model (19) can be obtained as

$$
H(\mathrm{j} \omega)=\frac{g_{n}(\mathrm{j} \omega)}{g_{d}(\mathrm{j} \omega)}
$$

where

$$
\left\{\begin{array}{l}
g_{n}(\mathrm{j} \omega)=\theta_{(0,1)}(\xi) \exp (-\mathrm{j} \omega \Delta t)+\theta_{(0,2)}(\xi) \exp (-\mathrm{j} 2 \omega \Delta t) \\
\quad+\theta_{(0,3)}(\xi) \exp (-\mathrm{j} 3 \omega \Delta t) \\
g_{d}(\mathrm{j} \omega)=1-\theta_{(1,0)}(\xi) \exp (-\mathrm{j} \omega \Delta t) \\
-\theta_{(2,0)}(\xi) \exp (-\mathrm{j} 2 \omega \Delta t)
\end{array}\right.
$$

The resonance of model (19) can be obtained by solving

$$
\begin{gathered}
\left.\frac{\mathrm{d}\left[g_{d}(\mathrm{j} \omega) g_{d}{ }^{*}(\mathrm{j} \omega)\right]}{\mathrm{d} \omega}\right|_{\omega=2 \pi f r d}=\mathrm{j} \Delta t \\
\times\left[\begin{array}{l}
\theta_{(1,0)}(\xi) \exp \left(-\mathrm{j} 2 \pi f_{r d} \Delta t\right) \\
-\theta_{(1,0)}(\xi) \exp \left(\mathrm{j} 2 \pi f_{r d} \Delta t\right) \\
+2 \theta_{(2,0)}(\xi) \exp \left(-\mathrm{j} 4 \pi f_{r d} \Delta t\right) \\
-2 \theta_{(2,0)}(\xi) \exp \left(\mathrm{j} 4 \pi f_{r d} \Delta t\right) \\
+\theta_{(1,0)}(\xi) \theta_{(2,0)}(\xi) \exp \left(\mathrm{j} 2 \pi f_{r d} \Delta t\right) \\
-\theta_{(1,0)}(\xi) \theta_{(2,0)}(\xi) \exp \left(-\mathrm{j} 2 \pi f_{r d} \Delta t\right)
\end{array}\right]=0
\end{gathered}
$$

where "*" is the complex conjugate. The output amplitude at the resonant frequency can be computed to find another design constraint

$$
\begin{aligned}
& \left|Y\left(\mathrm{j} f_{r d}\right)\right|=\left|H\left(\mathrm{j} 2 \pi f_{r d}\right) U\left(\mathrm{j} 2 \pi f_{r d}\right)\right| \\
& \quad=\left|\begin{array}{c}
\theta_{(0,1)}(\xi)+\theta_{(0,2)}(\xi) \exp (-\mathrm{j} \omega \Delta t) \\
+\theta_{(0,3)}(\xi) \exp (-\mathrm{j} 2 \omega \Delta t) \\
1-\theta_{(1,0)}(\xi) \exp (-\mathrm{j} \omega \Delta t) \\
-\theta_{(2,0)}(\xi) \exp (-\mathrm{j} 2 \omega \Delta t)
\end{array}\right| \leq 3.5 \times 10^{-5} \mathrm{~m}
\end{aligned}
$$

Many different sets of designs can satisfy the design requirements of (24) and (25). For example, a design can be determined as

$$
F_{0}=3 \mathrm{~N}, \mu=0.29, K_{T}=0.8 \times 10^{5} \mathrm{~N} / \mathrm{mm} ; N_{0}=6 \mathrm{~N}
$$

such that

$$
f_{r d}=105.5 \mathrm{~Hz},\left|Y\left(\mathrm{j} f_{r d}\right)\right|=3.08 \times 10^{-5} \mathrm{~m}
$$

A comparison of the model (19) predicted and true output spectra of the system under design (26) is shown in Fig.11, which clearly indicates the system output spectrum is basically as expected by the design.

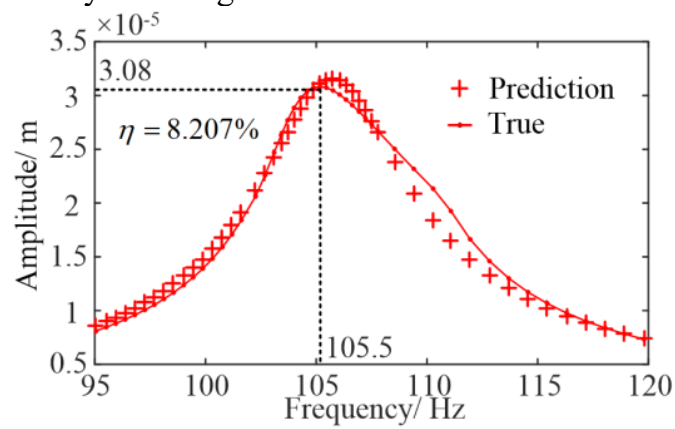

FIGURE 11: VALIDATION OF THE DESIGN RESULTS

\section{CONCLUSIONS}

The analysis and design of gas turbine fan blades with dovetail roots are computationally expensive due to strong nonlinearities and complex physics involved in the dovetail. There is a high demand in industries to develop efficient methods to improve the design of such complex systems. In order to solve this problem, a data driven surrogate model is built for the blade system, based on which the analysis and design of the blade system can be carried out. The surrogate model, known as the NP-ARX model, is a linear input-output model with the model coefficients represented by a nonlinear function of the design parameters of interest. A new frequency domain identification algorithm is proposed to determine the NP-ARX model by using harmonic input output data under different sets of the design parameters to address the highly redundant issues with using traditional time domain model identification approaches. By using the NP-ARX model, the system FRF can be evaluated and used for the analysis and design of the blade system. A case study based on a high fidelity FE simulation of a blade system with frictional roots is used to demonstrate the application of the data driven surrogate model based design. From the results of this study, it can be concluded that by using the data driven surrogate model based design method, an efficient and accurate design of aero-engine fan systems can be achieved. The approach would be extended to solve the analysis and design problems of many other complex systems. Further works will involve experimental studies and using the experimental data to conduct surrogate modelling and the model based system design. 


\section{APPENDIX}

Tab. A The coefficients of the surrogate model for the first vibration mode

\begin{tabular}{|c|c|c|c|c|c|}
\hline & $y(k-1)$ & $y(k-2)$ & $u(k-1)$ & $u(k-2)$ & $u(k-3)$ \\
\hline 1 & $\begin{array}{l}-0.5732 \\
\times 10^{-4} \\
\end{array}$ & $\begin{array}{l}-0.2638 \\
\times 10^{-5}\end{array}$ & - & $\begin{array}{l}-0.9791 \\
\times 10^{-9} \\
\end{array}$ & $\begin{array}{l}0.4955 \\
\times 10^{-13} \\
\end{array}$ \\
\hline$F_{0}$ & - & $\begin{array}{l}-0.9156 \\
\times 10^{-8}\end{array}$ & - & - & - \\
\hline$\mu$ & - & $\begin{array}{l}0.4988 \\
\times 10^{-6}\end{array}$ & - & - & - \\
\hline$K_{T}$ & - & $\begin{array}{l}0.5553 \\
\times 10^{-11} \\
\end{array}$ & - & - & - \\
\hline$N_{0}$ & $\begin{array}{l}-0.3967 \\
\times 10^{-5}\end{array}$ & $\begin{array}{l}0.1143 \\
\times 10^{-7} \\
\end{array}$ & - & - & - \\
\hline$F_{0}^{2}$ & $\begin{array}{l}0.6709 \\
\times 10^{-6} \\
\end{array}$ & $\begin{array}{l}0.1028 \\
\times 10^{-8} \\
\end{array}$ & - & $\begin{array}{l}-0.4534 \\
\times 10^{-11} \\
\end{array}$ & - \\
\hline$F_{0} \mu$ & $\begin{array}{l}-0.1074 \\
\times 10^{-4} \\
\end{array}$ & $\begin{array}{l}-0.4998 \\
\times 10^{-8}\end{array}$ & - & - & - \\
\hline$F_{0} K_{T}$ & $\begin{array}{l}-0.3741 \\
\times 10^{-10} \\
\end{array}$ & $\begin{array}{l}-0.2229 \\
\times 10^{-12} \\
\end{array}$ & - & $\begin{array}{l}0.2701 \\
\times 10^{-15} \\
\end{array}$ & - \\
\hline$F_{0} N_{0}$ & $\begin{array}{l}-0.5308 \\
\times 10^{-6}\end{array}$ & $\begin{array}{l}0.4281 \\
\times 10^{-9} \\
\end{array}$ & - & $\begin{array}{l}0.8139 \\
\times 10^{-12} \\
\end{array}$ & - \\
\hline$\mu^{2}$ & - & $\begin{array}{l}-0.4472 \\
\times 10^{-6} \\
\end{array}$ & - & - & - \\
\hline$\mu K_{T}$ & $\begin{array}{l}-0.5018 \\
\times 10^{-9} \\
\end{array}$ & $\begin{array}{l}-0.1683 \\
\times 10^{-11}\end{array}$ & - & - & - \\
\hline$\mu N_{0}$ & $\begin{array}{l}0.1930 \\
\times 10^{-4} \\
\end{array}$ & $\begin{array}{l}0.2091 \\
\times 10^{-7}\end{array}$ & - & $\begin{array}{l}0.1590 \\
\times 10^{-10} \\
\end{array}$ & - \\
\hline$K_{T}^{2}$ & - & $\begin{array}{l}-0.2897 \\
\times 10^{-16} \\
\end{array}$ & - & - & - \\
\hline$N_{0} K_{T}$ & $\begin{array}{l}0.1301 \\
\times 10^{-10} \\
\end{array}$ & $\begin{array}{l}0.1336 \\
\times 10^{-12} \\
\end{array}$ & - & - & - \\
\hline$N_{0}^{2}$ & - & $\begin{array}{l}-0.1705 \\
\times 10^{-8}\end{array}$ & - & - & - \\
\hline
\end{tabular}

\section{ACKNOWLEDGEMENTS}

This work was supported by the UK EPSRC. We are also grateful to the support of Rolls-Royce plc through Vibration University Technology Centre at Imperial College London.

\section{REFERENCES}

[1] Zhu, Y. P., Luo, Z., Zhao, X., \& Han, Q. Determination method of the structure size interval of dynamically similar models for predicting vibration characteristics of the coated thin plates. Proceedings of the Institution of Mechanical Engineers, Part C: Journal of Mechanical Engineering Science, 229(1), 59-68, 2015.

[2] Zhang, D., Fu, J., Zhang, Q., \& Hong, J. An effective numerical method for calculating nonlinear dynamics of structures with dry friction: application to predict the vibration response of blades with underplatform dampers. Nonlinear Dynamics, 88(1), 223-237, 2017.

[3] Krack, M., Salles, L., \& Thouverez, F. Vibration prediction of bladed disks coupled by friction joints. Archives of Computational Methods in Engineering, 24(3), 589-636, 2017.
[4] He, B., Ouyang, H., Ren, X., \& He, S. Dynamic response of a simplified turbine blade model with under-platform dry friction dampers considering normal load variation. Applied Sciences, 7(3), 228, 2017.

[5] Sun, Y., Yuan, J., Pesaresi, L., \& Salles, L. Nonlinear Vibrational Analysis for Integrally Bladed Disk Using Frictional Ring Damper. In Journal of Physics: Conference Series (Vol. 1106, No. 1, p. 012026). IOP Publishing, 2018.

[6] Fouvry, S., and Paulin, C. An effective friction energy density approach to predict solid lubricant friction endurance: Application to fretting wear. Wear, 319 (1-2), 211-226, 2014.

[7] Barman, K., Shipway, P., Voisey, K., and Pattinson, G. The role of a thermally sprayed cuniin underlayer in the durability of a dry-film lubricant system in fretting-a phenomenological model. Tribology International, 123 , 307-315, 2018.

[8] Yuan, J., Scarpa, F., Allegri, G., Titurus, B., Patsias, S., \& Rajasekaran, R. Efficient computational techniques for mistuning analysis of bladed discs: a review. Mechanical Systems and Signal Processing, 87, 71-90, 2017.

[9] Yuan, J., El-Haddad, F., Salles, L., \& Wong, C. Numerical Assessment of Reduced Order Modeling Techniques for Dynamic Analysis of Jointed Structures With Contact Nonlinearities. Journal of Engineering for Gas Turbines and Power, 141(3), 031027, 2019.

[10] Yuan, J., Salles, L., Wong, C. and Patsias, S. A Novel Penalty-Based Reduced Order Modelling Method for Dynamic Analysis of Joint Structures. In IUTAM Symposium on Model Order Reduction of Coupled Systems, Stuttgart, Germany, May 22-25, 165-176, 2020. Springer, Cham.

[11] Brunton, S. L., Proctor, J. L., \& Kutz, J. N. Discovering governing equations from data by sparse identification of nonlinear dynamical systems. Proceedings of the National Academy of Sciences, 113(15), 3932-3937, 2016.

[12] Kibangou, A. Y., \& Favier, G. Wiener-Hammerstein systems modeling using diagonal Volterra kernels coefficients. IEEE signal processing letters, 13(6), 381-384, 2006.

[13] Billings, S. A. Nonlinear system identification: NARMAX methods in the time, frequency, and spatio-temporal domains. John Wiley \& Sons, 2013.

[14] Zhu, Y. P., \& Lang, Z. Q. Design of nonlinear systems in the frequency domain: an output frequency response function-based approach. IEEE Transactions on Control Systems Technology, 26(4), 1358-1371, 2017.

[15] Zhu, Y. P., \& Lang, Z. Q. The effects of linear and nonlinear characteristic parameters on the output frequency responses of nonlinear systems: The associated output frequency response function. Automatica, 93, 422427, 2018.

[16] Adetona, O., Garcia, E., \& Keel, L. H. A new method for the control of discrete nonlinear dynamic systems using neural networks. IEEE Transactions on Neural Networks, 
11(1), 102-112, 2000.

[17] Pesaresi, L., Salles, L., Jones, A., Green, J. S., \& Schwingshackl, C. W. Modelling the nonlinear behaviour of an underplatform damper test rig for turbine applications. Mechanical Systems and Signal Processing, 85, 662-679, 2017

[18] Liang, Y. Z., Fang, K. T., \& Xu, Q. S. Uniform design and its applications in chemistry and chemical engineering. Chemometrics and Intelligent Laboratory Systems, 58(1), 43-57, 2001.

[19] Stein, M. Large sample properties of simulations using Latin hypercube sampling. Technometrics, 29(2), 143-151, 1987.

[20] Wei, H. L., \& Billings, S. A. Constructing an overall dynamical model for a system with changing design parameter properties. International Journal of Modelling Identification and Control, 5(2), 93-104, 2008.

[21] Liu, H., Zhu, Y. P., Luo, Z., \& Han, Q. PRESS-based EFOR algorithm for the dynamic parametrical modeling of nonlinear MDOF systems. Frontiers of Mechanical Engineering, 13(3), 390-400, 2018

[22] Jones, J. P., \& Billings, S. A. Recursive algorithm for computing the frequency response of a class of non-linear difference equation models. International Journal of Control, 50(5), 1925-1940, 1989.

[23] Winker, P., \& Fang, K. T. Application of thresholdaccepting to the evaluation of the discrepancy of a set of points. SIAM Journal on Numerical Analysis, 34(5), 20282042, 1997.

[24] http://www.math.hkbu.edu.hk/UniformDesign/

[25] Fang, K. T., Lin, D. K., Winker, P., \& Zhang, Y. Uniform design: theory and application. Technometrics, 42(3), 237248, 2000.

[26] Fang, K. T., \& Ma, C. X. Wrap-around L2-discrepancy of random sampling, Latin hypercube and uniform designs. Journal of complexity, 17(4), 608-624, 2001. 